\title{
Development of a Wireless Temperature Sensor Using Polymer-Derived Ceramics
}

\author{
Ran Zhao, ${ }^{1}$ Gang Shao, ${ }^{2} \mathrm{Ni} \mathrm{Li},{ }^{3}$ Chengying $\mathrm{Xu},{ }^{4}$ and Linan $\mathrm{An}^{1}$ \\ ${ }^{1}$ Department of Materials Science and Engineering, Advanced Materials Processing and Analysis Center, \\ University of Central Florida, Orlando, FL 32816, USA \\ ${ }^{2}$ School of Materials Science and Engineering, Zhengzhou University, Zhengzhou, Henan 450001, China \\ ${ }^{3}$ Department of Mechanical and Aerospace Engineering, University of Central Florida, Orlando, FL 32816, USA \\ ${ }^{4}$ Department of Mechanical Engineering, Florida State University, Tallahassee, FL 32310, USA
}

Correspondence should be addressed to Ni Li; ni.li@ucf.edu and Linan An; lan@mail.ucf.edu

Received 18 January 2016; Revised 16 March 2016; Accepted 29 March 2016

Academic Editor: Gyuhae Park

Copyright (c) 2016 Ran Zhao et al. This is an open access article distributed under the Creative Commons Attribution License, which permits unrestricted use, distribution, and reproduction in any medium, provided the original work is properly cited.

A temperature sensor has been developed using an embedded system and a sensor head made of polymer-derived SiAlCN ceramics (PDCs). PDC is a promising material for measuring high temperature and the embedded system features low-power consumption, compact size, and wireless transmission. The developed temperature sensor has been experimentally tested to demonstrate the possibility of using such sensors for real world applications.

\section{Introduction}

Accurate temperature measurements are crucial for many applications, such as chemical processing, power generation, and engine monitoring. As a result, development of temperature sensors has always been a focus of microsensor field. A variety of materials have been studied for temperature sensor applications, for example, semiconducting silicon and silicon carbide. Silicon based sensors are typically used at temperatures lower than $350^{\circ} \mathrm{C}$ due to accelerated material degradation at higher temperature $[1,2]$. Silicon carbide based sensors are better than silicon based sensors in high temperature measurement and can be applied in temperatures up to $500^{\circ} \mathrm{C}[3-5]$.

Polymer-derived SiAlCN ceramics (PDCs) are another widely studied material that demonstrate properties such as excellent high temperature stability [6] as well as good oxidation/corrosion resistance [7]. PDCs have been considered as a promising material for measuring high temperature [8]. Our early works have showed that PDC sensor head can accurately measure high temperature up to $830^{\circ} \mathrm{C}$ [9] using data acquisition system from National Instruments. The cost and size of the sensor system must be significantly reduced before it can be deployed for real world applications. In this paper, we develop a temperature sensor using PDC and an embedded system. Comparing to the National Instruments data acquisition equipment used in the previous paper, the newly developed embedded sensor is much smaller $\left(9.7 \mathrm{dm}^{3}\right.$ versus $\left.0.3 \mathrm{dm}^{3}\right)$, lighter $(5.97 \mathrm{~kg}$ versus $0.19 \mathrm{~kg}$ ), and cheaper (approximately $\$ 8000$ versus $\$ 170$ ). A WiFi module is also added so the temperature measurement can be transmitted wirelessly. The embedded board and WiFi module used in this paper are commercially available. The experiments in this paper demonstrate the possibility of deploying PDC based sensors for real world applications.

\section{Fabrication of the PDC Sensor Head}

In this study, the PDC sensor head is fabricated by following the procedure reported previously [9]. In brief, $8.8 \mathrm{~g}$ of commercially available liquid-phased polysilazane (HTT1800, Kion) and $1.0 \mathrm{~g}$ of aluminum-tri-sec-butoxide (ASB, SigmaAldrich) are first reacted together at $120^{\circ} \mathrm{C}$ for 24 hours under constant magnetic stirring to form the liquid precursor for SiAlCN. The precursor is then cooled down to room temperature, followed by adding $0.2 \mathrm{~g}$ of dicumyl peroxide (DP) into the liquid under sonication for 30 minutes. DP is the thermal initiator which can lower the solidification 


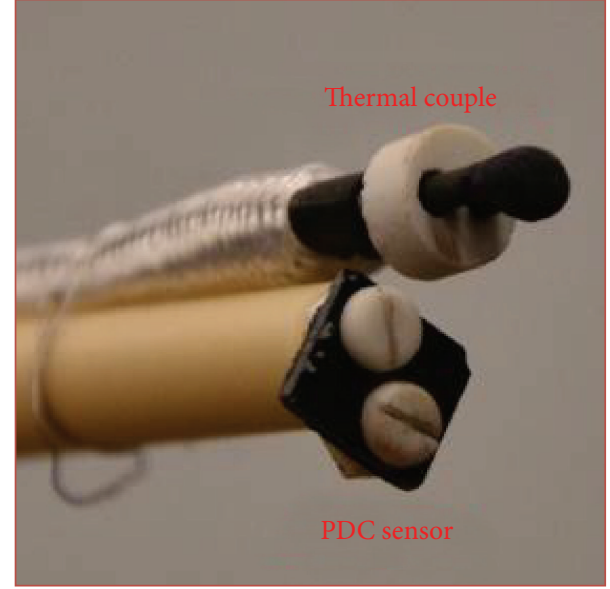

FIGURE 1: PDC sensor head, along with thermal couple for calibration and validation.

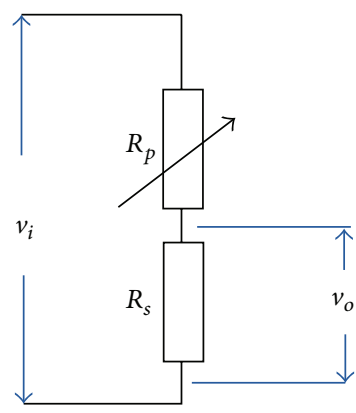

FIGURE 2: Diagram of resistance-to-voltage conversion circuit.

temperature and tailor the electrical properties [10]. The resultant liquid mixture is solidified by heat-treatment at $150^{\circ} \mathrm{C}$ for 24 hours. The disk-shaped green bodies are then prepared by ball-milling the solid into fine powder of $\sim 1 \mu \mathrm{m}$ and subsequently uniaxially pressing. A rectangular-shaped sample is cut from the discs and pyrolyzed at $1000^{\circ} \mathrm{C}$ for 4 hours. The entire fabrication is carried out in high-purity nitrogen to avoid any possible contamination.

Pt wires are attached to the sensor head by two ceramic fasteners on the two mounting holes on the diagonal of the sensor head. To improve the conductivity, both mounting holes are coated with Pt plasma; see Figure 1.

\section{Sensor Design and Characterization}

3.1. Resistance Conversion Circuit. PDC sensors are resistive sensors whose resistance must be converted to voltage to be picked up by analog to digital converter (ADC). A simple circuit as shown in Figure 2 is designed to serve that purpose. The sensor head is connected in series with a metal film resistor, denoted as $R_{s}$, and we have the following equation:

$$
R_{p}=\frac{v_{i}-v_{o}}{v_{o}} R_{s}
$$

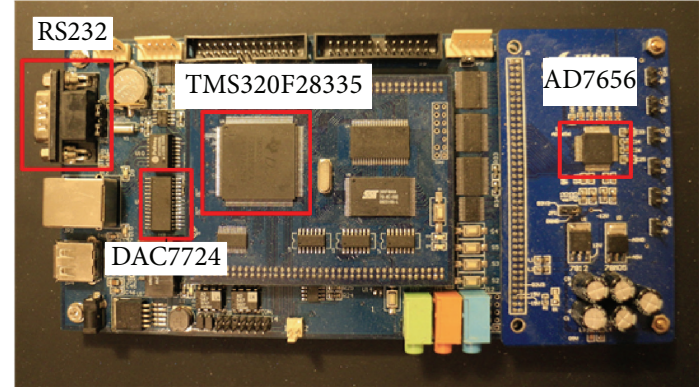

FIgURE 3: Embedded experiment board.

where $v_{o}$ is the output voltage of the circuit, $R_{p}$ represents the PDC sensor resistance, and $v_{i}$ is the voltage supplied to the circuit. In this paper, a resistor of $R_{s}=0.998 \mathrm{k} \Omega$ is used.

3.2. Design of Core of the PDC Sensor. A YX-F28335 embedded experimental board from YanXu (http://www .adianji.com/) is used to build the sensor system. The board is equipped with one 16-bit ADC (AD7656 from Analog Device) and one 12-bit digital-analog converter (DAC7724 from Texas Instruments). The picture of the embedded board is shown in Figure 3 with important components highlighted in red boxes. The core of the system is a TMS320F28335 microcontroller from Texas Instruments. TMS320F28335 is a high performance floating-point microcontroller of Texas Instruments' C2000 microcontroller family.

To measure temperature using the PDC sensor, the processor needs to perform the following tasks: (1) supply voltage $v_{i}$ to the circuit through DAC7724; (2) sample the circuit output $v_{o}$ using AD7656 and convert the output to temperature measurement; and (3) transmit data to readers from the RS232 port.

The input signal $v_{i}$ to the conversion circuit is a sinusoidal signal of $\pm 10 \mathrm{~V}$. The sinusoidal signal can bypass the parasitic capacitor in series to the PDC probe. The noise from the furnace coil can also be greatly subdued. The sensor output voltage $v_{o}$ is approximately sinusoidal as well and its magnitude can be computed using Fast Fourier Transformation (FFT) or curve fitting using recursive least square method (RLSM) [11]. Comparing to FFT, RLSM is more computationally efficient but may have numerical instability because TMS320F28335 only supports IEEE 754 floating-point arithmetic. Here we prefer FFT for fast prototyping purpose because Texas Instruments provides FPU library that performs floating FFT routines on C2000 series microcontroller. Next we explain how the sensor works.

A high-priority interrupt service request (ISR1) based on a CPU timer continues reading a look-up-table and drives the DAC7724 to generate the input signal $v_{i}$. The frequency of $v_{i}$ is controlled by the frequency of ISR1. ISR1 also samples circuit output from AD7656 and adds the data to a 1024-point buffer if there is no FFT running. Once the buffer is filled up, ISR1 stops writing the buffer and the FFT routine starts. The FFT routine is implemented in another slower low-priority interrupt service (ISR2). Once the FFT routine is completed, ISR2 will give ISR1 the permission to clean and write the 


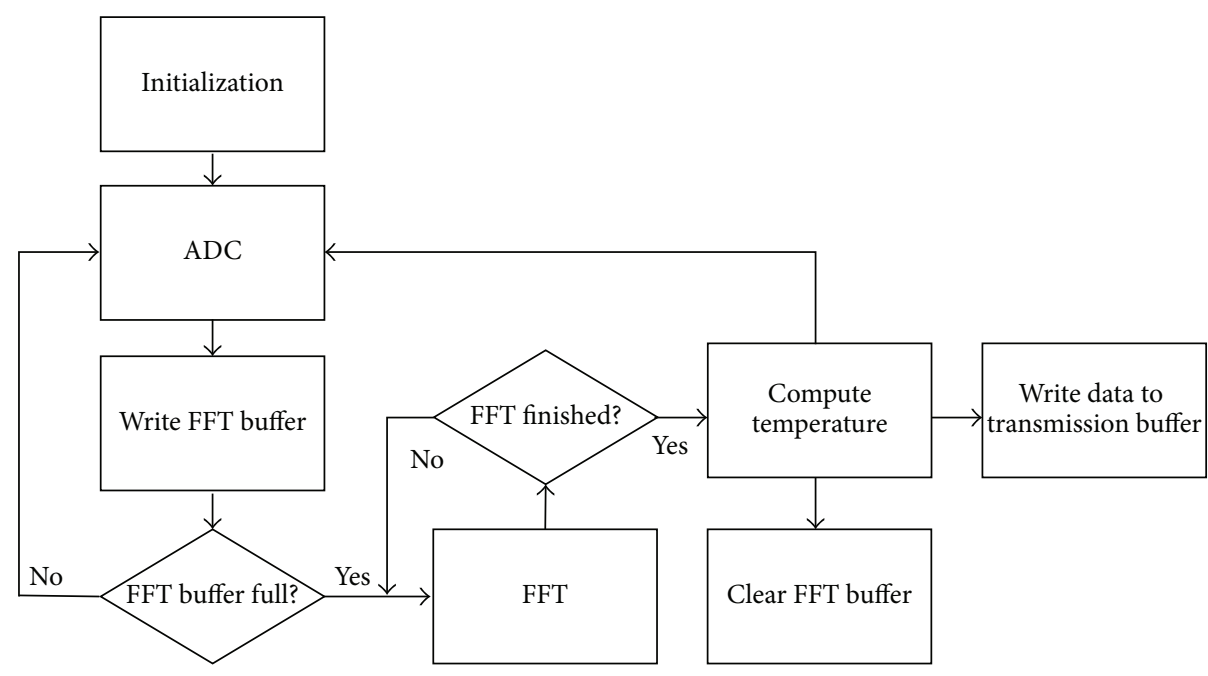

FIgURE 4: Software flowchart.

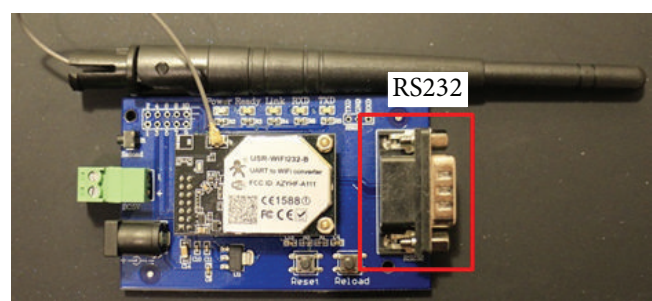

Figure 5: WiFi module.

input buffer again. The magnitude from the FFT is used as the circuit output $v_{o}$. The software flowchart is shown in Figure 4.

3.3. Wireless Signal Transmission. A WiFi module from Youren Tech (http://www.usr.cn/) shown in Figure 5 is connected to the embedded board through a RS-232 port. The computed temperature is written to a data register and then transmitted by the WiFi module automatically. The WiFi module can be wirelessly accessed as a hotspot. Once connected, readers (e.g., PC computers, hand-hold devices) can directly access the data from a virtual serial port simulated by the driver that comes with the WiFi module.

3.4. Auxiliary Measuring System Using Thermal Couple. A Ktype thermal couple is used to provide the reference temperature inside the furnace tube, which is used to characterize and verify the performance of the PDC sensor.

\section{Results and Discussion}

4.1. Sensor Characterization. The temperature-resistance curves of the PDC sensor head were obtained in the furnace setup shown in Figure 6.

The tube furnace (GSL-1100x from MTI) is programmed to raise temperature from $500^{\circ} \mathrm{C}$ to $850^{\circ} \mathrm{C}$ and then cool back down to $500^{\circ} \mathrm{C}$. To get the steady state measurement of the sensor, the temperature was set to dwell at several plateaus

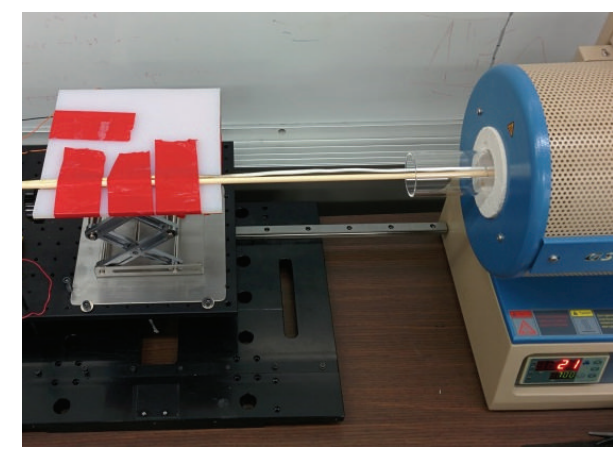

FIGURE 6: Experimental setup for sensor characterization.

during the test and the temperature difference between two consecutive plateaus is $50^{\circ} \mathrm{C}$. The furnace program was run twice to check the repeatability of the PDC sensor head. The reference temperature and the output of the PDC sensor are both plotted in Figure 7, where the blue solid line and red dashed line are data from first and second runs, respectively. It is clear that PDC sensor demonstrates good repeatability as the voltage outputs from the two tests are very close.

It should be noted that the reference temperatures used in this paper are not from the furnace but from the external thermal couple placed next to the PDC sensor (Figure 1). This is because the furnace (GSL-1100x from MTI) we used does not have temperature data recording option. The temperature measured by the external thermal couple is about $25^{\circ} \mathrm{C}$ lower than the readings of the furnace. This measurement difference is consistent and therefore does not affect the sensor calibrations and performance validations.

The resistance of the sensor head should comply with the Thermistor equation [12]:

$$
\ln \frac{1}{R_{p}}=c_{1} \frac{1}{T}-c_{2},
$$

where $c_{1}$ and $c_{2}$ are constant coefficients, which are experimentally determined using linear optimization. The result 


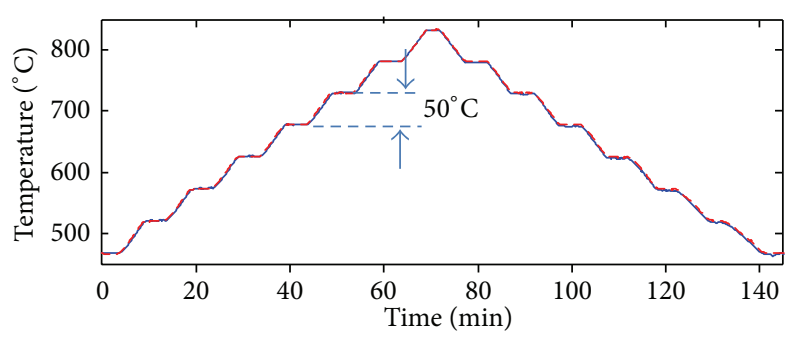

(a)

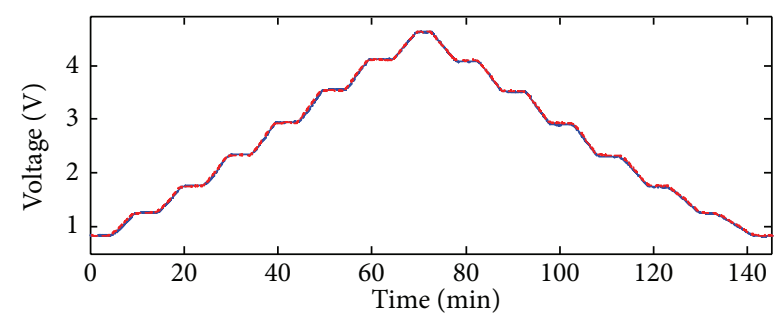

(b)

Figure 7: (a) Temperature measured by thermal couple and (b) voltage output $v_{o}$ from the PDC sensor.

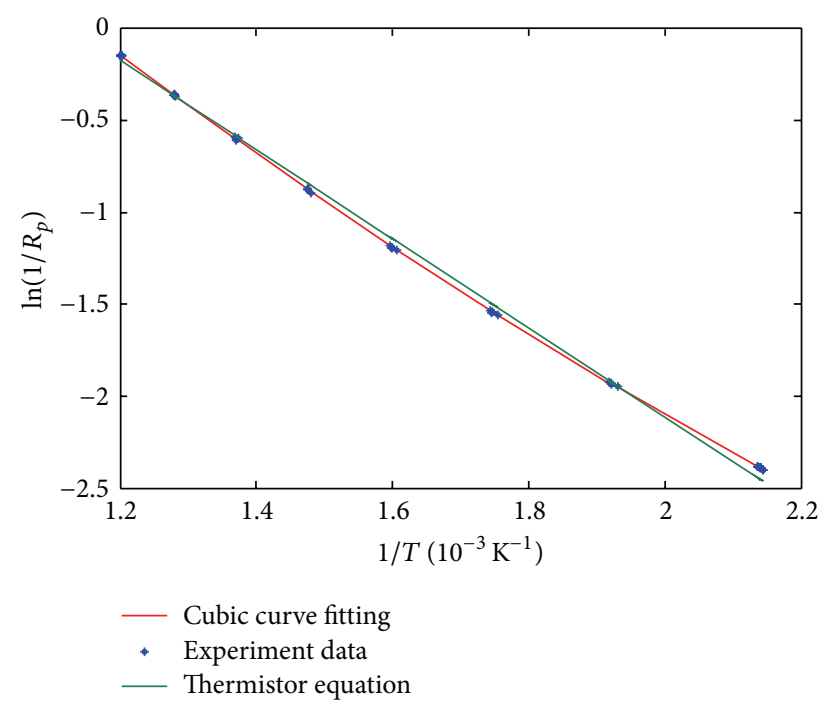

FIGURE 8: Sensor resistance versus temperature in a format of $\ln \left(1 / R_{p}\right)$ versus $1 / T$.

of the curve fitting is shown in Figure 8 as the green curve.

It is clear that $\ln \left(1 / R_{p}\right)$ is only approximately linear to the inverse of temperature and the linear curve fitting is not perfect. To get better results over the entire temperature range, we use a third-order polynomial to replace (2):

$$
c_{1}\left(\ln \frac{1}{R_{p}}\right)+c_{2}\left(\ln \frac{1}{R_{p}}\right)^{2}+c_{3}\left(\ln \frac{1}{R_{p}}\right)^{3}+c_{4}=\frac{1}{T},
$$

where $c_{1}, c_{2}, c_{3}$, and $c_{4}$ are constant coefficients and the result is shown in Figure 8 as the red curve. Apparently, the thirdorder polynomial matches the experiment results better than the linear curve fitting. With the coefficients determined by

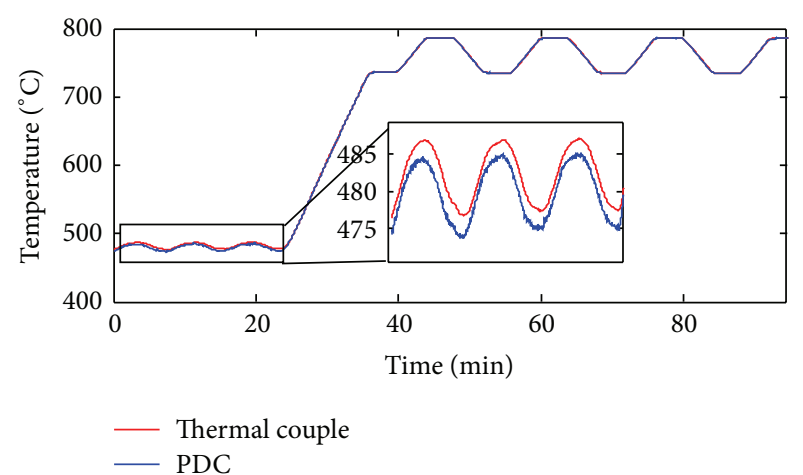

(a)

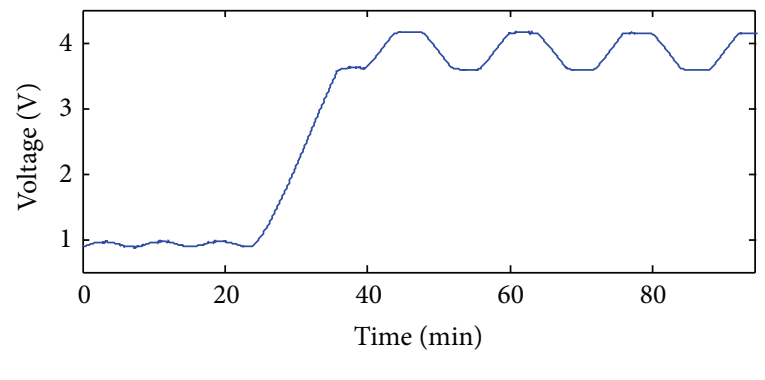

- PDC

(b)

FIGURE 9: Sensor performance validation. (a) Temperatures measured by thermal couple and PDC. (b) Raw voltage signal from PDC circuit.

the numerical optimization, the temperature measurements can be obtained by combining (1) and (3).

4.2. Performance Validation. In this section, we proceed to examine the performance of the sensor. To comprehensively test the performance, two furnace programs are designed. The low temperature program first commands the furnace to dwell at $510^{\circ} \mathrm{C}$ for 2 minutes and then rise to $520^{\circ} \mathrm{C}$ in 2 minutes. After that, the program stays at $520^{\circ} \mathrm{C}$ for 2 minutes and then goes back to $510^{\circ} \mathrm{C}$ in 2 minutes. The high temperature program first stays at $750^{\circ} \mathrm{C}$ for 5 minutes, rises to $800^{\circ} \mathrm{C}$ in 5 minutes, stays at $800^{\circ} \mathrm{C}$ for 5 minutes, and then cools back down to $750^{\circ} \mathrm{C}$ in 5 minutes.

The validation test repeats the low temperature program 3 times and then rises to $750^{\circ} \mathrm{C}$ in 12 minutes and then repeats the high temperature program 3 times. The furnace program is designed to cover both low and high temperatures as well as different temperature change rates. The experimental results are shown in Figure 9. The raw voltage signal shown in Figure 9(b) is used to calculate the corresponding temperature measurement which is compared to data from the external thermal couple shown in Figure 9(a). It is clear that the PDC sensor system can accurately measure temperature at both low and high temperatures. The maximum error is about $2.5^{\circ} \mathrm{C}$ at low temperature range. This measurement error can be further reduced by fine tuning the calibration experiments and by using better curve fittings that characterize the sensor better. 


\section{Conclusion}

In this paper, a temperature sensor was successfully fabricated using polymer-derived SiAlCN ceramics and an embedded system. Characterization and validation experiments demonstrate excellent performance. More importantly, the low cost and compact size of the sensor make it possible to deploy for actual application.

Our research efforts have also been devoted to building PDC sensors using low-power consumption MSP430 from Texas Instruments and radio frequency identification (RFID) technology to develop battery-less sensors. The results would be presented in our future publications.

\section{Competing Interests}

The authors declare that they have no competing interests.

\section{Authors' Contributions}

Ran Zhao and Gang Shao contributed equally to this work.

\section{References}

[1] M. Biebl, G. Brandl, and R. T. Howe, "Young's modulus of in situ phosphorus-doped polysilicon," in Proceedings of the 8th International Conference on Solid-State Sensors and Actuators, and Eurosensors IX, pp. 80-83, Stockholm, Sweden, Jun 1995.

[2] G. L. Pearson, W. T. Read Jr., and W. L. Feldmann, "Deformation and fracture of small silicon crystals," Acta Metallurgica, vol. 5, no. 4, pp. 181-191, 1957.

[3] E. A. de Vasconcelos, S. A. Khan, W. Y. Zhang, H. Uchida, and T. Katsube, "Highly sensitive thermistors based on high-purity polycrystalline cubic silicon carbide," Sensors and Actuators A: Physical, vol. 83, no. 1-3, pp. 167-171, 2000.

[4] J. B. Casady, W. C. Dillard, R. W. Johnson, and U. Rao, "A hybrid $6 \mathrm{H}-\mathrm{SiC}$ temperature sensor operational from $25^{\circ} \mathrm{C}$ to $500^{\circ}$ C, IEEE Transactions on Components, Packaging, and Manufacturing Technology: Part A, vol. 19, no. 3, pp. 416-422, 1996.

[5] J. Yang, "A silicon carbide wireless temperature sensing system for high temperature applications," Sensors (Switzerland), vol. 13, no. 2, pp. 1884-1901, 2013.

[6] R. Riedel, A. Kienzle, W. Dressier, L. Ruwisch, J. Bill, and F. Aldinger, "A silicoboron carbonitride ceramic stable to 2,000 C)" Nature, vol. 382, no. 6594, pp. 796-798, 1996.

[7] Y. Wang, W. Fei, and L. An, "Oxidation/corrosion of polymerderived SiAlCN ceramics in water vapor," Journal of the American Ceramic Society, vol. 89, no. 3, pp. 1079-1082, 2006.

[8] H. Ryu, Q. Wang, and R. Raj, "Ultra-high temperature semiconductors made from polymer-derived ceramics," Journal of the American Ceramic Society, vol. 93, no. 6, pp. 1668-1676, 2010.

[9] R. Zhao, G. Shao, Y. Cao, L. An, and C. Xu, “Temperature sensor made of polymer-derived ceramics for high-temperature applications," Sensors and Actuators A: Physical, vol. 219, pp. 5864, 2014.

[10] Y. Wang, L. Zhang, W. Xu et al., "Effect of thermal initiator concentration on the electrical behavior of polymer-derived amorphous silicon carbonitrides," Journal of the American Ceramic Society, vol. 91, no. 12, pp. 3971-3975, 2008.
[11] L. Ljung and T. Söderström, Theory and Practice of Recursive Identification, MIT Press, 1983.

[12] F. J. Hyde, Thermistors, Iliffe Books, London, UK, 1971. 


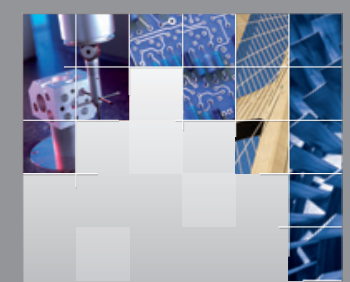

\section{Enfincering}
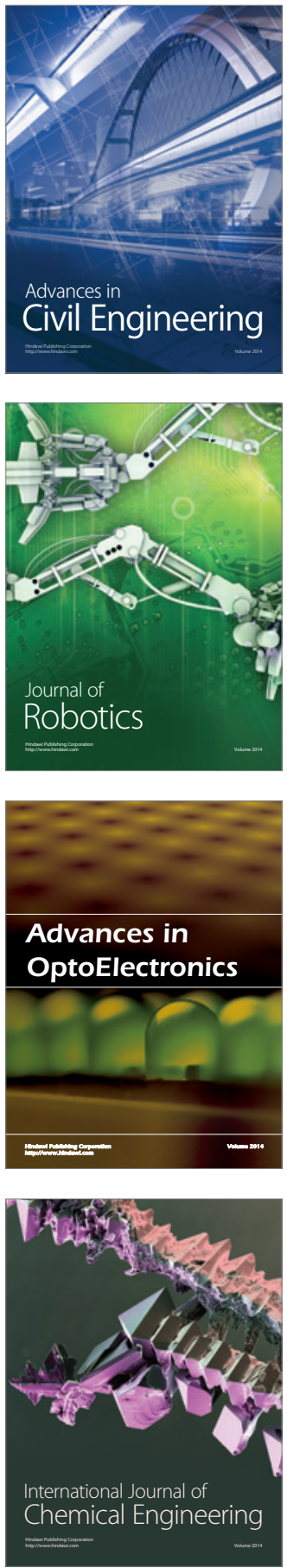

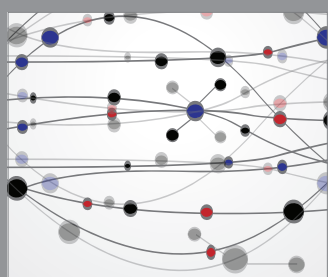

The Scientific World Journal

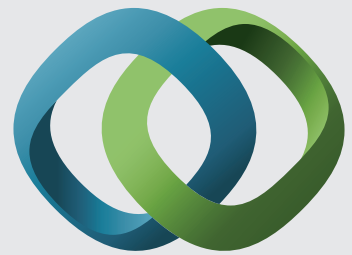

\section{Hindawi}

Submit your manuscripts at

http://www.hindawi.com
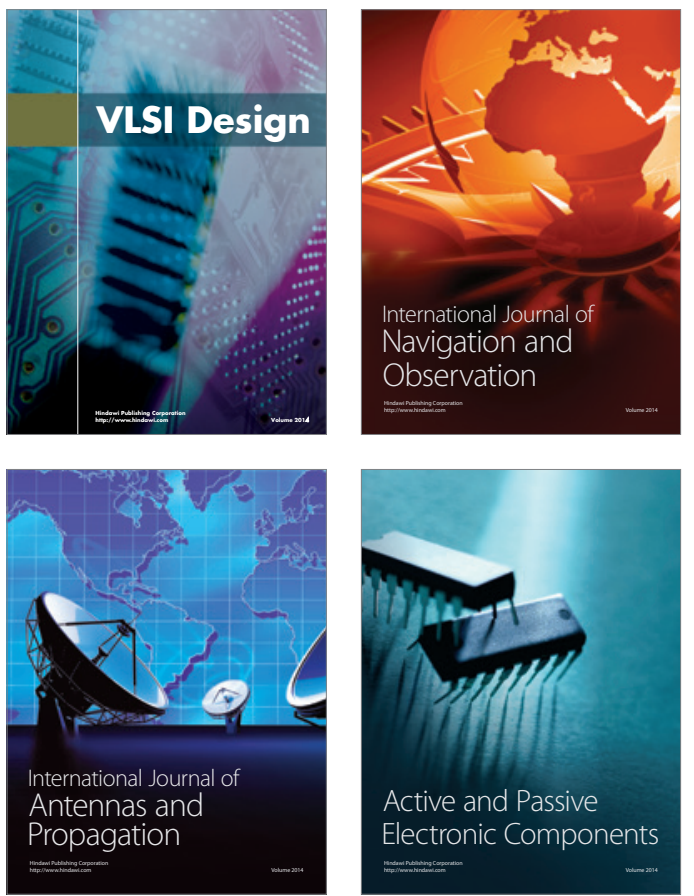
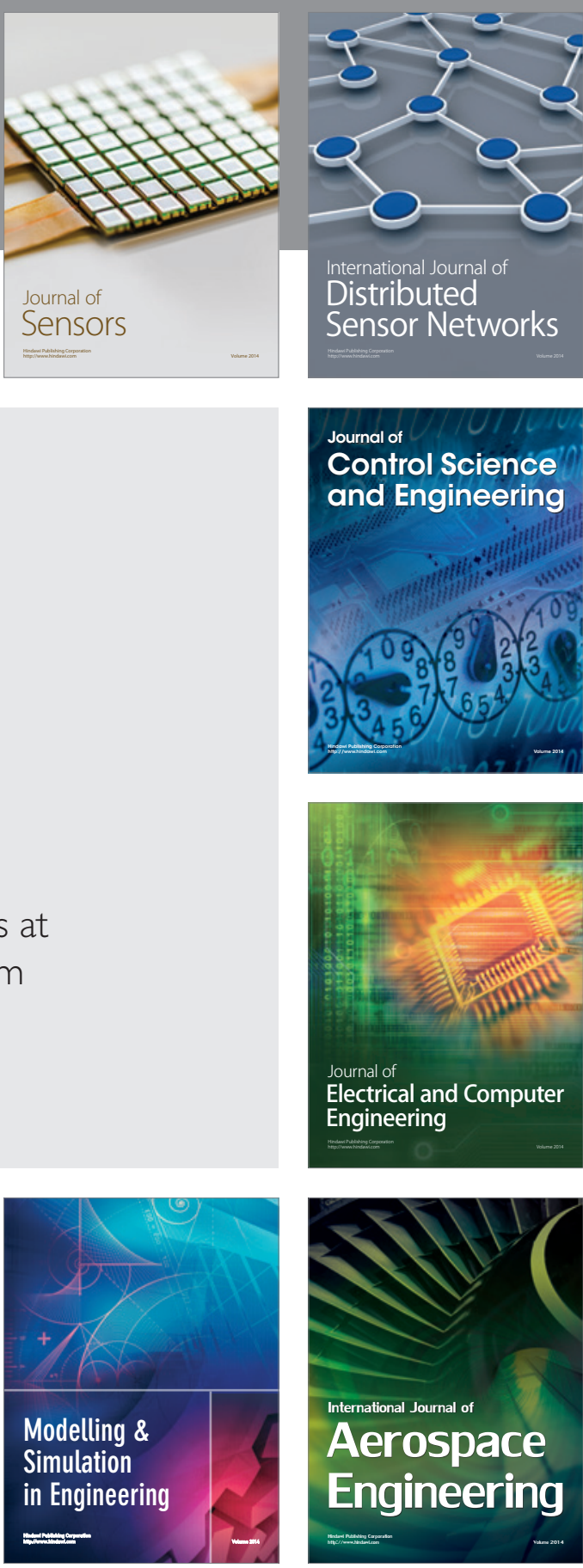

International Journal of

Distributed

Sensor Networks

Journal of

Control Science

and Engineering
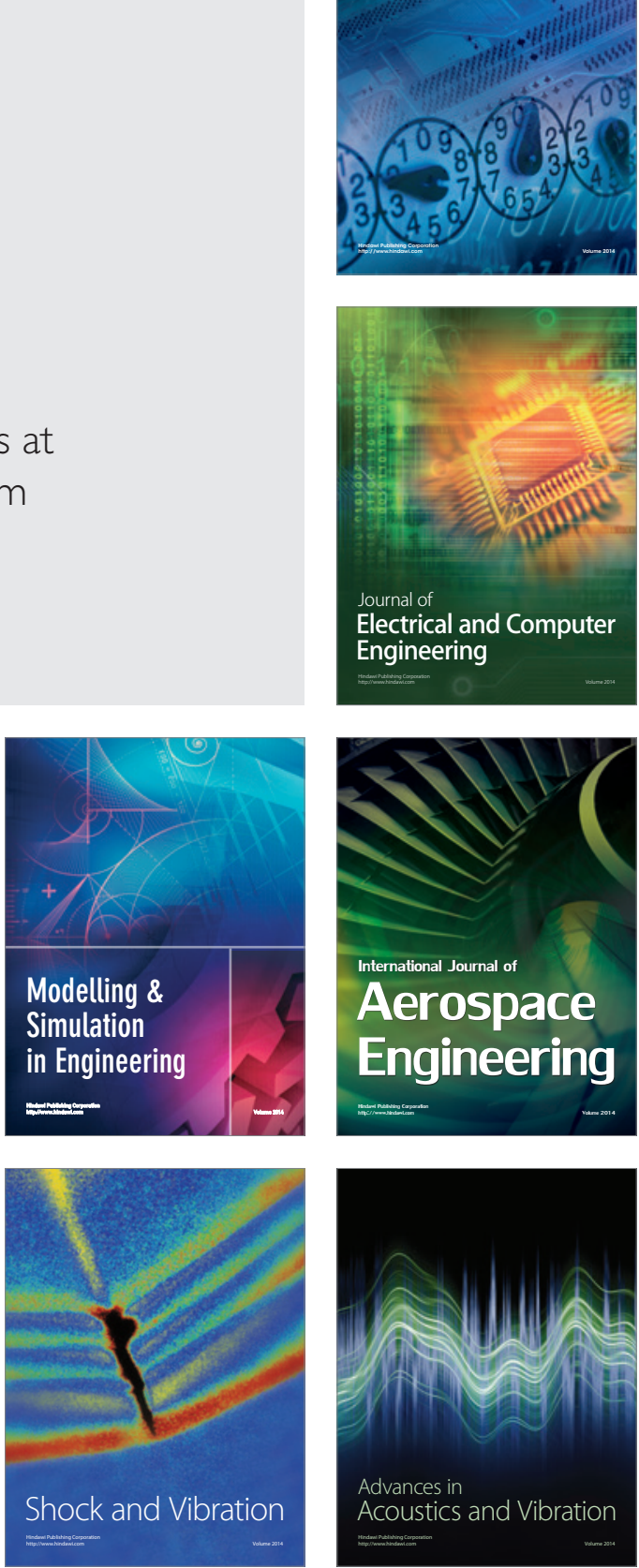